\title{
Definition of recurrence of pelvic organ prolapse: is it really important?
}

\author{
Stefano Salvatore • Gabriele Siesto • Diaa E. E. Rizk
}

Received: 19 February 2011 / Accepted: 22 March 2011 / Published online: 12 April 2011

(C) The International Urogynecological Association 2011

The beginning of wisdom is the definition of terms.

\section{Socrates}

Pelvic organ prolapse (POP) is becoming an important women's health priority worldwide because the cumulative lifetime risk for undergoing surgical intervention is estimated to be greater than $11 \%[1,2]$. Almost one third of these women will develop postoperative recurrence requiring further surgery, thus representing a significant public health problem [3]. Causes of recurrence of POP are poorly understood but include younger or older age, obesity, genetic predisposition for weakness of the pelvic floor, advanced prolapse, and performing an inappropriate primary procedure [4-6]. Although the etiology of recurrence of POP is frequently discussed in the literature, very few studies report on the outcome of surgical treatment in women with recurrent disease. In the published case series to date, data on the long-term outcome are often lacking or difficult to compare and therefore the cumulative surgical cure rate

\section{S. Salvatore $(\square)$}

Department of Obstetrics and Gynecology, University of Insubria, Piazza Biroldi 1,

21100 Varese, Italy

e-mail: stefanosalvatore@hotmail.com

\section{G. Siesto}

Department of Obstetrics and Gynecology,

Humanitas Clinical Institute,

Rozzano, Milan, Italy

D. E. E. Rizk

Department of Obstetrics and Gynecology, Faculty of Medicine, Ain Shams University,

Cairo, Egypt and/or independent efficacy of each surgical technique remain uncertain $[3,7]$. This is usually attributed to the use of various and non-validated tools to measure the outcome of a large number of operative techniques, particularly the novel disposable surgical kits, in a small subset of patient cohorts. The lack of a uniformly accepted definition of recurrence including a detailed description of the anatomical location of the defect and whether this is in the same or different vaginal compartment may also be responsible [3, 6, 7].

It might therefore be helpful to have a standard definition and/or classification of recurrent POP as a separate clinicopathological entity from primary disease and are urgently needed by the urogynecologic community, similar to recent standardization reports [8]. This endeavor will increase the scientific validity of outcome measures of surgical intervention for recurrent POP with a potential for selecting the most effective procedures and will contribute to our understanding of the pathogenesis of recurrence. A standardized terminology of POP recurrence will also allow comparison of the results of surgical treatment between different surgeons and centers and will provide robust clinical evidence to assist in counseling women about the expected treatment outcome [9]. Hence, the ambiguous postoperative outcome measures that are currently used in the biomedical literature such as "surgical failure," "persistent," "recurrence," "relapse," or "de novo" should now be replaced by the appropriate medical terminology that facilitates interpretation of data obtained by different researchers in this field.

There is also mounting evidence at present that anatomical evaluation, per se, does not often reflect patient perception of their condition and women with the same preoperative POP stage may have completely different expectations [9]. Moreover, the concept of restoring normal vaginal anatomy in women with POP using reconstructive pelvic surgery is 
difficult to define since some postoperative "residual" vaginal descent can be completely asymptomatic. Should we then expect the same results when we operate on women with recurrent POP stage III or IV? Does this have an impact on defining our surgical success or failure? Is it important to consider the re-operation rate as the best indirect measure of patients' satisfaction when evaluating the global efficacy of surgical procedures for recurrent POP $[8,9]$ ?

Our last consideration is the need to include a thorough evaluation of urinary, bowel, and sexual dysfunctions in our pre-and postoperative assessment of the outcome of reconstructive pelvic surgery for recurrent POP. A true surgical cure, in fact, should not be considered as just an anatomical restoration of the prolapsed organ but also as an improvement of any subjective pelvic complaints noted before surgery for recurrent POP with the aim of improving the patients quality of life $(\mathrm{QoL})$ after the operation $[8,9]$. Finally, we are aware that the clinical evaluation of POP, whether primary or recurrent, and of its treatment represents a challenging issue especially with the growing prevalence of the disease [5]. The major difficulty is to find an individual score or stage that combines anatomical distortion, compartment dysfunction, and their impact on QoL in an individual subject. We believe that developing an instrument that is able to measure all these factors would help dramatically in tailoring interventions and monitoring the results of surgical treatment of recurrent POP and wonder whether creating a multi-site grading system similar to the TNM staging used in oncology could assist with this task. Until this system is available, we must first define our terms!
Conflicts of interest Stefano Salvatore has been a speaker for Promedon and Gynecare. The other authors have no interests to disclose.

\section{References}

1. Fialkow M, Newton K, Lentz G, Weiss N (2008) Lifetime risk of surgical management for pelvic organ prolapse or urinary incontinence. Int Urogynecol J 19:437-440

2. Fritel X, Varnoux N, Zins M et al (2009) Symptomatic pelvic organ prolapse at midlife, quality of life, and risk factors. Obstet Gynecol 113:609-616

3. Salvatore S, Siesto G, Serati M (2010) Risk factors for recurrence of genital prolapse. Curr Opin Obstet Gynecol 22:420-424

4. Salvatore S, Athanasiou S, Digesu GA et al (2009) Identification of risk factors for genital prolapse recurrence. Neurourol Urodynam 29:301-304

5. Wu JM, Hundley AF, Fulton RG, Myers ER (2009) Forecasting the prevalence of pelvic floor disorders in US women: 2010 to 2050. Obstet Gynecol 114:1278-1283

6. Diez-Itza I, Aizpitarte T, Becerro A (2007) Risk factors for the recurrence of pelvic organ prolapsed after vaginal surgery: a review at 5 years after surgery. Int Urogynecol J 18:13171324

7. Denman MA, Gregory WT, Boyles SH et al (2008) Reoperation 10 years after surgically managed pelvic organ prolapse and urinary incontinence. Am J Obstet Gynecol 198(555):e1-e5

8. Haylen BT, de Ridder D, Freeman RM et al (2010) An International Urogynecological Association (IUGA)/International Continence Society (ICS) joint report on the terminology for female pelvic floor dysfunction. Int Urogynecol J 21:5-26

9. Haliloglu B, Rizk DEE (2010) Subjective versus objective measurement of surgical outcomes of treatment of female stress urinary incontinence: it's not just black and white. Int Urogynecol J $21: 761-762$ 